\title{
Satellite Attitude Control System Design Taking into Account the Fuel Slosh and Flexible Dynamics
}

\author{
Alain G. de Souza ${ }^{1}$ and Luiz C. G. de Souza ${ }^{1,2}$ \\ ${ }^{1}$ National Institute for Space Research (INPE), Avenida dos Astronautas 1758, 12227-010 São José dos Campos, SP, Brazil \\ ${ }^{2}$ Brasília University, Campus Gama, Área Especial de Indústria-A-UnB, 72444-240 Brasilia, DF, Brazil \\ Correspondence should be addressed to Luiz C. G. de Souza; lcgadelha@gmail.com
}

Received 31 March 2014; Revised 23 July 2014; Accepted 29 July 2014; Published 10 September 2014

Academic Editor: Antonio F. Bertachini A. Prado

Copyright (c) 2014 A. G. de Souza and L. C. G. de Souza. This is an open access article distributed under the Creative Commons Attribution License, which permits unrestricted use, distribution, and reproduction in any medium, provided the original work is properly cited.

\begin{abstract}
The design of the spacecraft Attitude Control System (ACS) becomes more complex when the spacecraft has different type of components like, flexible solar panels, antennas, mechanical manipulators and tanks with fuel. The interaction between the fuel slosh motion, the panel's flexible motion and the satellite rigid motion during translational and/or rotational manoeuvre can change the spacecraft center of mass position damaging the ACS pointing accuracy. This type of problem can be considered as a FluidStructure Interaction (FSI) where some movable or deformable structure interacts with an internal fluid. This paper develops a mathematical model for a rigid-flexible satellite with tank with fuel. The slosh dynamics is modelled using a common pendulum model and it is considered to be unactuated. The control inputs are defined by a transverse body fixed force and a moment about the centre of mass. A comparative investigation designing the satellite ACS by the Linear Quadratic Regulator (LQR) and Linear Quadratic Gaussian (LQG) methods is done. One has obtained a significant improvement in the satellite ACS performance and robustness of what has been done previously, since it controls the rigid-flexible satellite and the fuel slosh motion, simultaneously.
\end{abstract}

\section{Introduction}

The problems due to fluid-structure interaction (FSI) are crucial in the design of many engineering systems. They appear in the dynamic behaviour of offshore and marine structures, road, and railroad containers partially filled with a fluid [1]. In space applications the problem appears when the dynamics of a rigid satellite with liquid fuel inside interacts with flexible structures [2]. One notes these problems are more relevant when the slosh unactuated dynamics motion has to be simultaneously controlled together to the rigidflexible motion of the satellite [3]. In the rigid-flexible satellite attitude control system (ACS) design the knowledge of the interaction between fluid motion (slosh) and flexible structure dynamics is important because this interaction can damage the ACS pointing requirements. A space structure, like rockets, geosynchronous satellites, and the space station, usually contains liquid in tanks that can represent more than $40 \%$ of the initial mass of the system. As a result, the first step to design its ACS is to obtain a detailed dynamics model of the space structure. When the fuel tanks are only partially filled and suffer a transversal acceleration and/or rotational motion, large quantities of fuel move uncontrollably inside the tanks and generate the sloshing effects. It has been shown in [4] that the dynamics interaction between the fuel motion and the rigid and/or flexible body dynamics can result in some kind of control instability. For minimizing these effects the ACS must be designed using a robust control method in order to assure stability and good performance to achieve the attitude control system requirement [5]. The dynamics of rigid-flexible satellite with fuel tanks when subject to large angle manoeuvre is only captured by complex nonlinear mathematical model. Besides, the remaining flexible and/or liquid vibration can introduce a tracking error resulting in a minimum attitude acquisition time. The problem of designing satellite nonlinear controller for rigid satellite has been done in [6] using the state-dependent Riccati equation (SDRE) method which is able to deal with high 
nonlinear plants. Due to the complexity of modelling the fluid and/or flexible dynamic of the system it is common to use mechanical systems analogies that describe this dynamic. Besides, if one needs to know some physical parameters related with the slosh or the flexibility dynamics it is common to obtain them by experimental apparatus or some kind of estimating method such as Kalman filter [7]. In [8] a new technique to control the attitude of a rigid-flexible satellite has been developed where a reaction wheel was used for controlling the angular motion and the vibrations are damped by piezoelectric patches that are symmetrically bonded in the panel's surfaces. A multiobjective approach has been used in [9] to solve the problem of optimal flexible solar sail trajectories control. A similar investigation has been done in [10] where, besides the design of the controller considering the effects of the interaction between the liquid motion (slosh) and the rigid dynamics, the parameters of the fuel slosh dynamics modelled by a simple pendulum are identified using the Kalman filter technique.

\section{Satellite Model with Slosh}

The phenomenon of sloshing is due to the movement of a free surface of a liquid that partially fills a compartment and this movement is oscillating. It depends on shape of the tank, the acceleration of gravity, and the axial/rotational acceleration of the tank. As representative of the behaviour of the total weight of the system it is accepted that when the mass of the liquid oscillates the mass center of the rigid body also oscillates, thereby disturbing the rigid-flexible part of the vehicle under consideration. As an oscillating movement it is natural to consider the wave generated by the movement of the liquid as a stationary wave. Each mode of oscillation has a special feature of this phenomenon under study, and one observes, in a quantitative sense, how much mass is displaced. Among all the modes that cause the greatest disruption in the system are the first and second modes. Despite the fact that oscillation has lower frequency it is capable of resulting in violent shifting of the center of mass of the liquid creating an oscillation in the system as a role. The other oscillation modes act less aggressive and may not even vary with the position of its center of mass due to the symmetry of the wave which on average causes no displacement.

Due to its complexity, the sloshing dynamics is usually represented by mechanical equivalents that describes and reproduces faithfully the actions and reactions due to forces and torques acting on the system. The main advantage of replacing the fluid model with an equivalent oscillating model is simplifying the analysis of motion in the rigid body dynamics, compared to the fluid dynamics equations. Due to the complexity of establishing an analytical model for the fluid moving freely within a closed tank, it is used as a simplified system, taking into account the following criteria [5]: (a) small displacements, (b) a rigid tank, and (c) no viscous, incompressible, and homogeneous liquid. Under these conditions the dynamics of the sloshing can be approximated by mechanical system consisting of a massspring or pendulum.
Consider a rigid spacecraft moving in a fixed plane, with a spherical fuel tank and including the lowest frequency slosh mode. Based on the Lagrange equation and the Rayleigh dissipation function one can model systems using the mechanical mass-spring and pendulum type system, respectively. Figure 1 shows a satellite model where slosh dynamics is represented by its pendulum analogous mechanical system.

The mass of the satellite and the moment of inertia, regardless of the fuel, are given by $m$ and $I$, respectively, and the mass equivalent of fuel and its inertia moment are given by $M_{f}$ and $I_{f}$, respectively. It is assumed as a transverse force $f$ and a pitching moment $M$. The constant thrust $F$ is responsible for the orbital transfer of the spacecraft with respect to the inertial reference system $(x, y, z)$ and it acts on the center of mass of spacecraft in the longitudinal axis. Also it is given the velocity of the center of the fuel tank $v_{x}$, $v_{z}$ and the attitude angle $\theta$ of the spacecraft with respect to a fixed reference $(X, Y, Z)$. Besides, one assumes as generalized coordinates that $V$ represents the linear velocity, $\omega$ represents the angular velocity of the rigid body, $a$ is the length of the pendulum rod, $b$ is the distance from satellite center of mass to the pendulum connected point, and $\psi$ is the angle of the pendulum with respect to the spacecraft longitudinal axis, which is assumed in the equilibrium position $\psi=0$ about the reference axis. The parameters $m_{f}, \psi$, and $a$ depend on the shape of the tank, chemical-physical characteristics of the fuel, and the fill ratio of the fuel tank.

The satellite equations of motion for the satellite with sloshing can be derived using the Lagrange equations given by

$$
\begin{gathered}
\frac{d}{d t}\left(\frac{\partial L}{\partial V}\right)+\omega^{\times} \frac{\partial L}{\partial V}=\tau_{t}, \\
\frac{d}{d t}\left(\frac{\partial L}{\partial \omega}\right)+\omega^{\times} \frac{\partial L}{\partial \omega}+V^{\times} \frac{\partial L}{\partial V}=\tau_{r}, \\
\frac{d}{d t}\left(\frac{\partial L}{\partial \dot{\psi}}\right)-\frac{\partial L}{\partial \psi}+\frac{\partial R}{\partial \dot{\psi}}=0,
\end{gathered}
$$

where $L$ is the Lagrangian of the system, $R$ is the Rayleigh dissipation function, $\tau_{r}$ is the internal torque, and $\tau_{t}$ is the external torque. Assume that $R, \tau_{r}, \tau_{t}, \omega$, and $V$ are given by

$$
\begin{gathered}
R=\frac{1}{2} \varepsilon \dot{\psi}^{2} ; \quad V=\left[\begin{array}{c}
v_{x} \\
0 \\
v_{z}
\end{array}\right] ; \quad \omega=\left[\begin{array}{l}
0 \\
\dot{\theta} \\
0
\end{array}\right] ; \\
\tau_{t}=\left[\begin{array}{c}
F \\
0 \\
f
\end{array}\right] ; \quad \tau_{r}=\left[\begin{array}{c}
0 \\
M+f b \\
0
\end{array}\right] .
\end{gathered}
$$

The position vector of the satellite mass center with respect to the inertial system is

$$
\vec{r}=(x-b) i+z \hat{k} .
$$




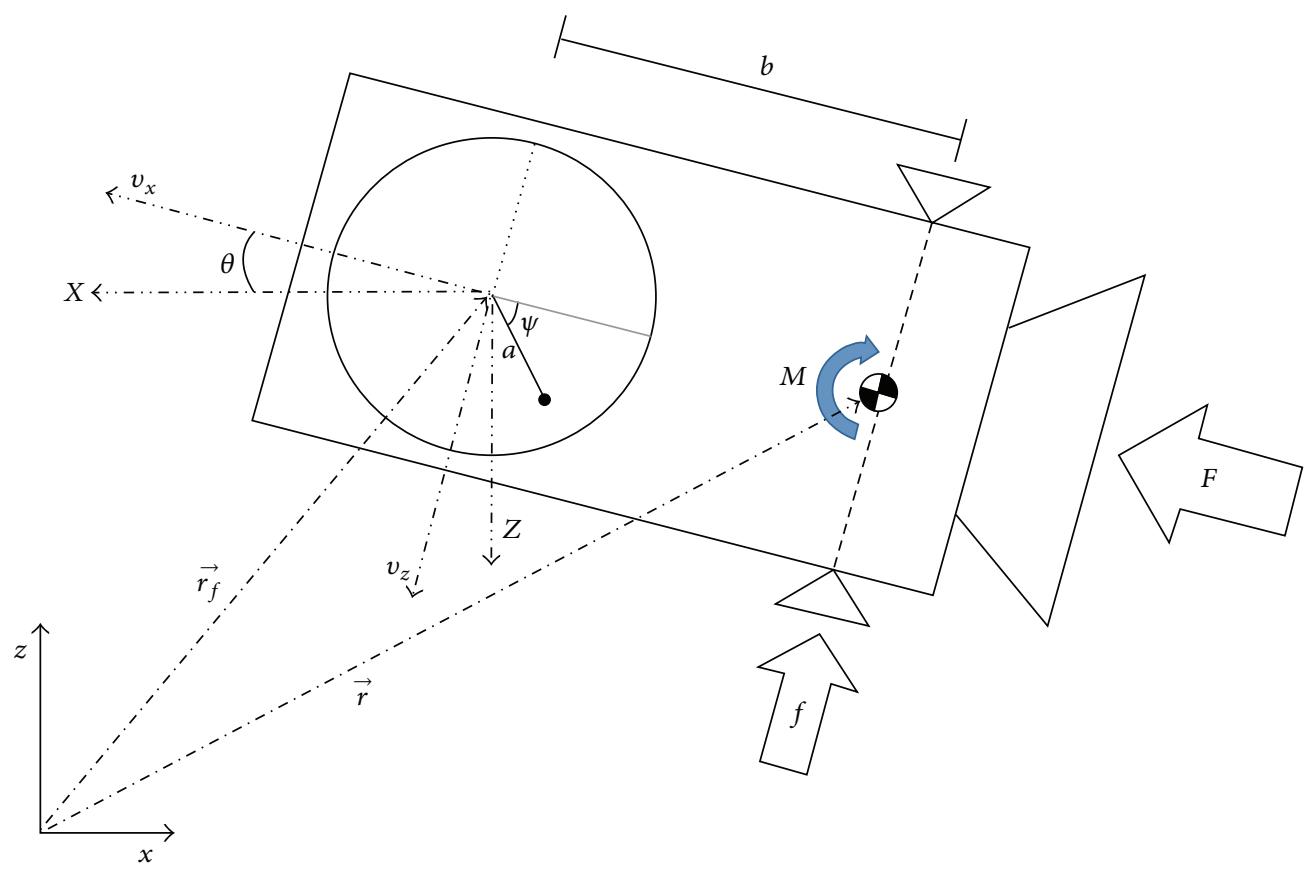

FIGURE 1: Satellite model with slosh dynamics pendulum analogous mechanical system.

Assuming the relations $v_{x}=\dot{x}+z \dot{\theta}$ and $v_{z}=\dot{z}-x \dot{\theta}$ the satellite velocity is given by

$$
\dot{\vec{r}}=v_{x} \hat{i}+\left(v_{z}+b \dot{\theta}\right) \hat{k}
$$

The position of the mass of fuel is given by

$$
\vec{r}_{f}=(x-a \cos (\psi)) \hat{i}+(z+a \operatorname{sen}(\psi)) \hat{k} .
$$

As a result, the velocity of the fuel mass is

$\dot{\vec{r}}_{f}=\left(v_{x}+a \operatorname{sen}(\psi)(\dot{\theta}+\dot{\psi})\right) \hat{i}+\left(v_{z}+a \cos (\psi)(\dot{\theta}+\dot{\psi})\right) \hat{k}$.

The Lagrangian of the entire system is given by

$$
L=\frac{1}{2} m \dot{\vec{r}}^{2}+\frac{1}{2} m_{f} \dot{\overrightarrow{\vec{r}}}_{f}^{2}+\frac{1}{2} I_{f}(\dot{\theta}+\dot{\psi})^{2}+\frac{1}{2} I \dot{\theta}^{2} .
$$

Substituting (4)-(6) into (7), using the relations given by (2), and performing the derivations of (1), one obtains the satellite equations of motion given by

$$
\begin{aligned}
\left(m+m_{f}\right)\left(\dot{v}_{x}+v_{z} \dot{\theta}\right)+m b \dot{\theta}+m_{f} a(\ddot{\psi}+\ddot{\theta}) \operatorname{sen}(\psi) \\
+m_{f} a(\dot{\theta}+\dot{\psi})^{2} \cos (\psi)=F
\end{aligned}
$$

$$
\begin{gathered}
\left(m+m_{f}\right)\left(\dot{v}_{z}-v_{x} \dot{\theta}\right)+m_{f} a(\ddot{\theta}+\ddot{\psi}) \cos (\psi) \\
-m_{f} a(\dot{\theta}+\dot{\psi})^{2} \operatorname{sen}(\psi) m b \ddot{\theta}=f \\
\left(I_{f}+m b^{2}\right) \ddot{\theta}+m b\left(\dot{v}_{z}-v_{x} \dot{\theta}\right)-\varepsilon \dot{\psi}=M+b f \\
\left(m_{f} a^{2}+I_{f}\right)(\ddot{\theta}+\ddot{\psi})+m_{f} a\left(\left(\dot{v}_{x}+v_{z} \dot{\theta}\right) \operatorname{sen}(\psi)\right. \\
\left.+\left(\dot{v}_{z}-v_{x} \dot{\theta}\right) \cos (\psi)\right)+\varepsilon \dot{\psi}=0 .
\end{gathered}
$$

Assuming the relations $a_{x}=\dot{v}_{x}+v_{z} \dot{\theta}, a_{z}=\dot{v}_{z}-v_{x} \dot{\theta}$ and substituting them into (8) and (9), one can isolate and obtain the satellite accelerations given by

$$
\begin{aligned}
& a_{x}=\frac{F-m b \dot{\theta}-m_{f} a(\ddot{\psi}+\ddot{\theta}) \operatorname{sen}(\psi)-m_{f} a(\dot{\theta}+\dot{\psi})^{2} \cos (\psi)}{m+m_{f}} \\
& a_{z}=\frac{f-m_{f} a(\ddot{\theta}+\ddot{\psi}) \cos (\psi)+m_{f} a(\dot{\theta}+\dot{\psi})^{2} \operatorname{sen}(\psi)-m b \ddot{\theta}}{m+m_{f}} .
\end{aligned}
$$

All equations derived previously are nonlinear. However, in order to design LQR and LQG controllers one has to get the linear set of equations of motion, which is obtained assuming that the system makes small movements around the zero point of equilibrium [10]. Now, substituting (12) into (10) and 


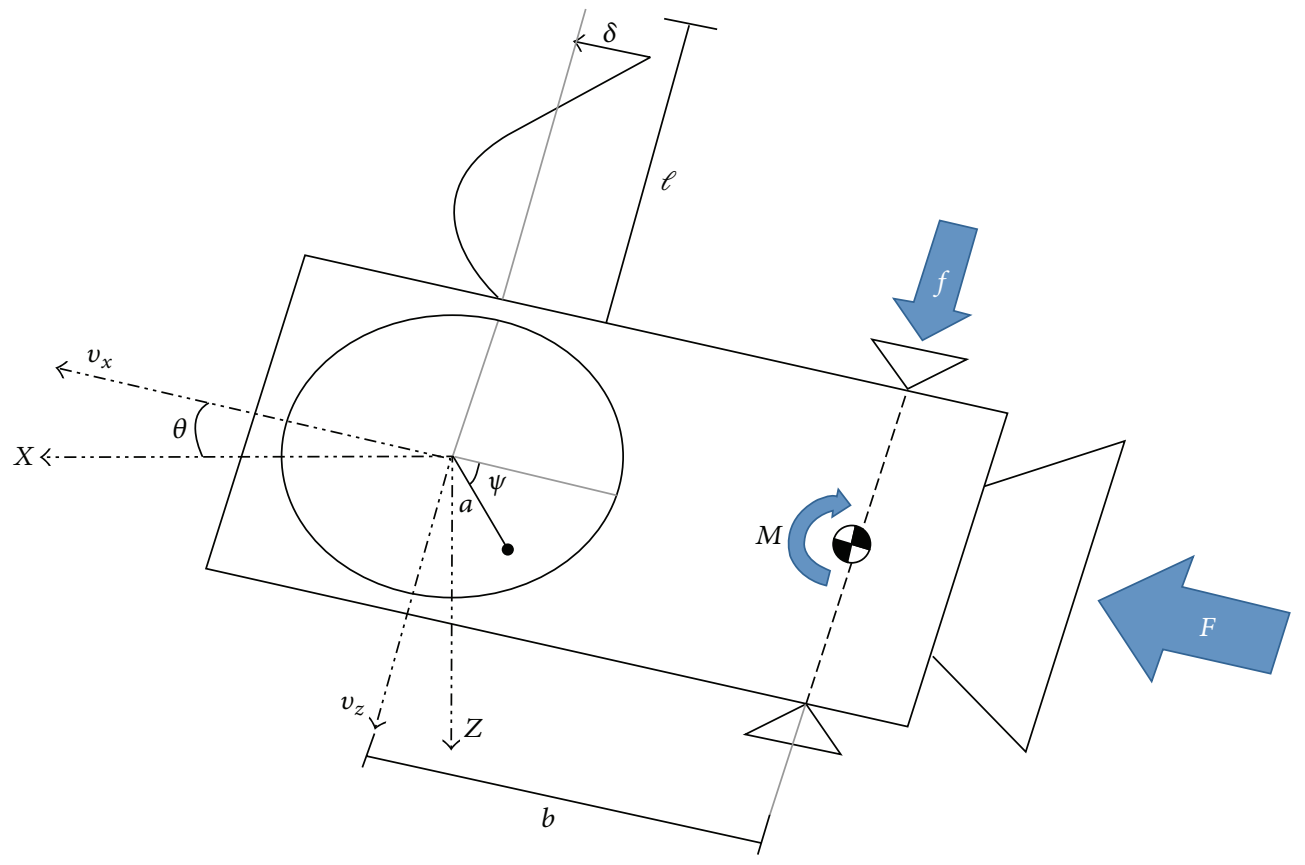

FIgURE 2: Satellite model with slosh dynamics and a flexible panel.

(11) and assuming the linearization conditions, one has the satellite equation of motion given by

$$
\begin{aligned}
& \begin{aligned}
\ddot{\theta}\left(I_{f}\right. & \left.+m^{*}\left(a^{2}-b a\right)\right)+\psi\left(I_{f}+m^{*} a^{2}\right)+a m_{f}^{*} F \psi+\varepsilon \dot{\psi} \\
& =-a m_{f}^{*} f
\end{aligned} \\
& \ddot{\theta}\left(I+m^{*}\left(b^{2}-b a\right)\right)-m^{*} a b \psi-\varepsilon \dot{\psi}=M+b^{*} f,
\end{aligned}
$$

where $b^{*}=b m_{f} /\left(m+m_{f}\right), m^{*}=m m_{f} /\left(m+m_{f}\right)$ and $m_{f}^{*}=$ $m_{f} /\left(m+m_{f}\right)$.

\section{Satellite Model with Sloshing and Flexibility}

To derive the equations of motion for the satellite model with sloshing and flexibility one considers the same rigid satellite with tank partially filled plus a flexible appendage connected to the satellite as shown in Figure 2.

The flexible appendage has mass $m_{p}$ and length $\ell$ and it has two motions, the angular motion $\theta$, resulting in a linear velocity $\ell \dot{\theta}$ and a flexible deformation $\delta$ with respect to the $z$-axis resulting in its variation given by $\dot{\delta}$. Thus, for small deformations, the panel velocity is given by

$$
v_{p}=\dot{\delta}+\ell \dot{\theta}
$$

The panel kinetic, potential energy and the dissipation function of energy $D$ are given by

$$
\begin{aligned}
T_{p} & =\frac{1}{2} m_{p}(\dot{\delta}+\ell \dot{\theta})^{2}, \\
E_{\text {potential }} & =\frac{\delta^{2}}{2} k, \\
D & =\frac{\dot{\delta}^{2}}{2} k_{d},
\end{aligned}
$$

where $k$ and $k_{d}$ are the panel elastic constant and the dissipation constant.

Now the Lagrangian considering the slosh and theappendices' flexibility is given by

$$
\begin{aligned}
L=\frac{1}{2}( & \left(m+m_{f}\right)\left(v_{x}^{2}+v_{z}^{2}\right)+m\left(2 v_{z} b \dot{\theta}+b^{2} \dot{\theta}^{2}\right) \\
+ & m_{f}(a(\dot{\theta}+\dot{\psi})(a(\dot{\theta}+\dot{\psi}) \\
& \left.\left.+2\left(v_{x} \operatorname{sen}(\psi)+v_{z} \cos (\psi)\right)\right)\right) \\
+ & \left.I \dot{\theta}^{2}+I_{f}(\dot{\theta}+\dot{\psi})^{2}+m_{p}\left(\dot{\delta}^{2}+2 \ell \dot{\theta} \dot{\delta}+\ell^{2} \dot{\theta}^{2}\right)-\delta^{2} k\right) .
\end{aligned}
$$

In order to obtain the equation of motion for the satellite with sloshing and flexible panel one uses (1) plus the Lagrange equation given by

$$
\frac{d}{d t}\left(\frac{\partial L}{\partial \dot{\delta}}\right)-\frac{\partial L}{\partial \delta}+\frac{\partial D}{\partial \dot{\delta}}=0
$$


by which after all derivation one obtains the nonlinear equations of motion given by

$$
\begin{gathered}
\left(m+m_{f}\right) a_{x}+m b \dot{\theta}^{2}+m_{f} a(\ddot{\psi}+\ddot{\theta}) \operatorname{sen}(\psi) \\
+m_{f} a(\dot{\theta}+\dot{\psi})^{2} \cos (\psi)=F \\
\left(m+m_{f}\right) a_{z}+m_{f} a(\ddot{\psi}+\ddot{\theta}) \cos (\psi) \\
-m_{f} a(\dot{\theta}+\dot{\psi})^{2} \operatorname{sen}(\psi)+m b \ddot{\theta}=f \\
\left(m b^{2}+I_{f}+m_{p} \ell^{2}\right) \ddot{\theta}+\ddot{\delta} m_{p} \ell+m b a_{z}-\varepsilon \dot{\psi}=M+f b \\
\left(m_{f} a^{2}+I_{f}\right)(\ddot{\psi}+\ddot{\theta})+m_{f} a\left(\operatorname{sen}(\psi) a_{x}+\cos (\psi) a_{z}\right) \\
+\varepsilon \dot{\psi}=0, \\
\ddot{\delta} m_{p}+\ddot{\theta} m_{p} \ell+\dot{\delta} k_{d}+\delta k=0 .
\end{gathered}
$$

In order to linearize the system of (18a), (18b), (18c), (18d), and (18e), one must first isolate $a_{x}$ and $a_{z}$ in (18a) and (18b) and replace them in (18c) and (18d). By doing this one obtains the system of linear equations given by

$$
\begin{gathered}
\ddot{\theta}\left(I+m_{p} \ell^{2}+m^{*}\left(b^{2}-b a\right)\right)+m_{p} \ell \ddot{\delta}-m^{*} a b \ddot{\psi} \\
-\varepsilon \dot{\psi}=M+b^{*} f \\
\ddot{\theta}\left(\frac{m^{*} b a}{I_{f}+m^{*} a^{2}}-1\right)=\ddot{\psi}+\dot{\psi}\left(\frac{\varepsilon}{I_{f}+m^{*} a^{2}}\right) \\
+\psi\left(\frac{a^{*} F}{I_{f}+m^{*} a^{2}}\right)+\frac{a^{*} f}{I_{f}+m^{*} a^{2}}, \\
-\ddot{\theta} \ell=\ddot{\delta}+\dot{\delta} \frac{k_{d}}{m_{p}}+\delta \frac{k}{m_{p}},
\end{gathered}
$$

where $m^{*}=m_{f} m /\left(m+m_{f}\right), a^{*}=m_{f} a /\left(m+m_{f}\right), b^{*}=$ $m_{f} b /\left(m+m_{f}\right)$.

\section{Linear Quadratic Gaussian (LQG)}

The LQG method is the union of the LQR problem with the Kalman filter problem. The separation principle [7] ensures that each problem can be solved independently of each other. However, if there is any state that is not available one uses the Kalman filter to estimate it in order to receive feedback.

Assume a plant described by the linear state equations given by

$$
\begin{aligned}
\dot{\mathbf{x}}(t) & =\mathbf{A} \mathbf{x}(t)+\mathbf{B} \mathbf{u}(t)+\Gamma w \\
\mathbf{y} & =\mathbf{C} \mathbf{x}(t)+v
\end{aligned}
$$

where $\mathbf{x}$ is the state vector, $\mathbf{A}$ is the state matrix, $\mathbf{B}$ is the input matrix, $\mathbf{y}$ is the output vector, $\mathbf{C}$ is the output matrix, $v$ and $w$ are white noise, and $\mathbf{u}$ is the control input. Following a similar approach described before, now the LQR gain is given by

$$
\mathbf{K}_{c}=\mathbf{R}^{-1} \mathbf{B}^{T} \mathbf{P}_{c}
$$

where $\mathbf{R}$ is real symmetric positive definite matrix and $\mathbf{P}_{c}$ is the symmetrical solution of the LQR Riccati equation given by

$$
\mathbf{A}^{T} \mathbf{P}_{c}+\mathbf{P}_{c} \mathbf{A}-\mathbf{P}_{c} \mathbf{B} \mathbf{R}^{-1} \mathbf{B}^{T} \mathbf{P}_{c}+\mathbf{M}^{T} \mathbf{Q M}=\mathbf{0} .
$$

Similarly the Kalman filter gain now is given by

$$
\mathbf{K}_{f}=\mathbf{P}_{f} \mathbf{C}^{T} \mathbf{V}^{-1}
$$

where $\mathbf{V}$ is real symmetric positive definite matrix and $\mathbf{P}_{f}$ is the symmetrical solution matrix of the KF Riccati equation given by

$$
\mathbf{P}_{f} \mathbf{A}^{T}+\mathbf{A} \mathbf{P}_{f}-\mathbf{P}_{f} \mathbf{C}^{T} \mathbf{V}^{-1} \mathbf{C} \mathbf{P}_{f}+\Gamma^{T} \mathbf{W} \Gamma=0
$$

where $\mathbf{P}_{c}=\mathbf{P}_{c}^{T} \geq 0$ and $\mathbf{P}_{f}=\mathbf{P}_{f}^{T} \geq 0$ and $\mathbf{Q}, \mathbf{R}, \mathbf{V}$, and $\mathbf{W}$ are weights matrices which can be regarded as setting parameters ("tuning") that must be manipulated until they find one acceptable response to the system. The LQG method is more realistic than the LQR method, since it can estimate the states that are not available for feedback and it allows including the noise in the model which represents imperfections of the system.

\section{Simulations Results}

The first simulation is the design comparison between the LQR and LQG control law, for the satellite model with sloshing dynamics given by (13a) and (13b). The parameters values used in the simulations are $m=600 \mathrm{Kg}, m_{f}=100 \mathrm{Kg}$, $I=720 \mathrm{Kg} / \mathrm{m}, I_{f}=90 \mathrm{Kg} / \mathrm{m}, a=0.3 \mathrm{~m}, b=0.3 \mathrm{~m}$, $F=500 \mathrm{~N}$, and $\varepsilon=0.19 \mathrm{Kgm}^{2} / \mathrm{s}$. The initial conditions used are $\theta=2^{\circ}, d \theta / d t=0.57^{\circ} / \mathrm{s}, \psi=1^{\circ}$, and $d \psi / d t=0^{\circ}$. Figure 3 shows that the LQR control law performance is better than the LQG, which indicates that considering the sloshing variables available for feedback helps the LQR control law, which is not true.

Figure 4 shows that both the torque and the force of the LQR controller are smaller than the LQG controller.

The second simulation is also the comparison between the LQR and LQG control law, but now the satellite model has the sloshing dynamics plus the flexible dynamics of panel, in which the data values are $m_{p}=10 \mathrm{~kg}, \ell=1.5 \mathrm{~m}$, $k=320 \mathrm{Kgrad}^{2} / \mathrm{s}^{2}$, and $k_{d}=0.48 \mathrm{Kgrad}^{2} / \mathrm{s}$. The simulations initials conditions are $\theta=2^{\circ}, d \theta / d t=0.57^{\circ} / \mathrm{s}, \psi=1^{\circ}$, $d \psi / d t=0^{\circ} / \mathrm{s}$, and $\delta=\dot{\delta}=0$.

Figure 5 shows that the LQR control law performance is better than the LQG only for controlling the angular motion. Once, for controlling the flexible motion, the LQR controller performance is damaged and it takes more time to damp the flexible motion. 


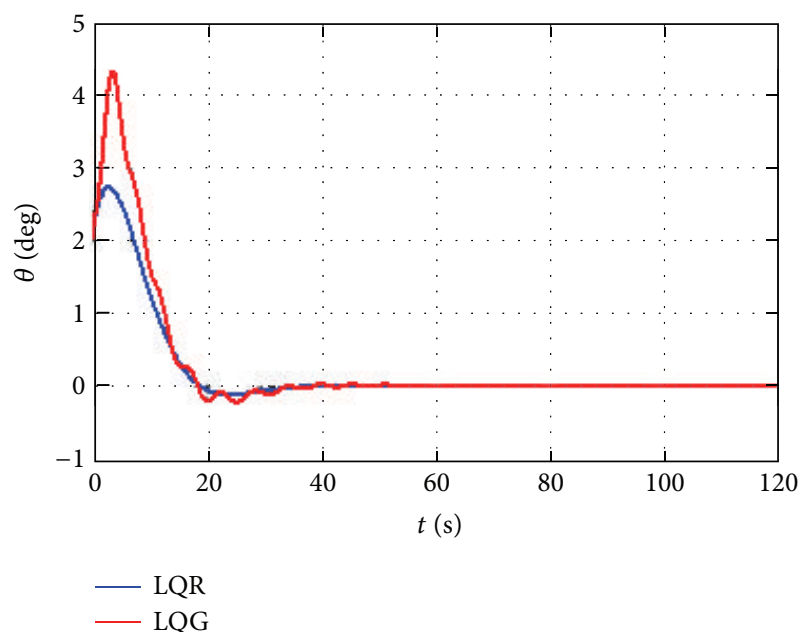

(a)

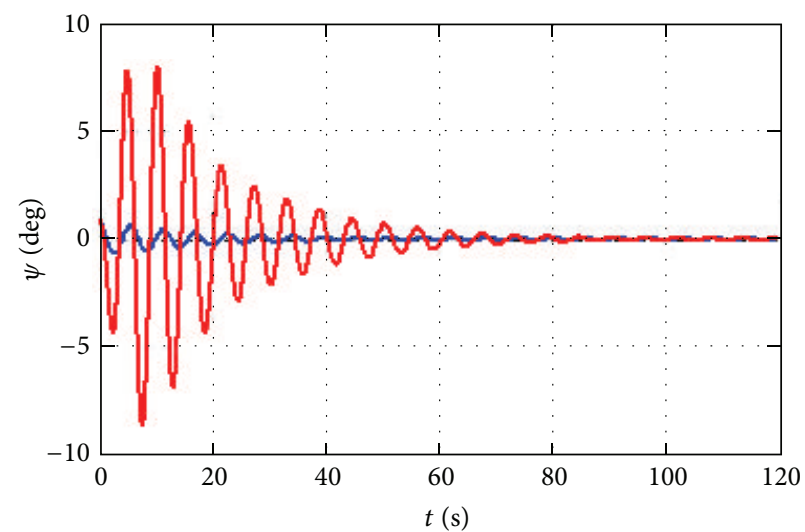

LQR
LQG

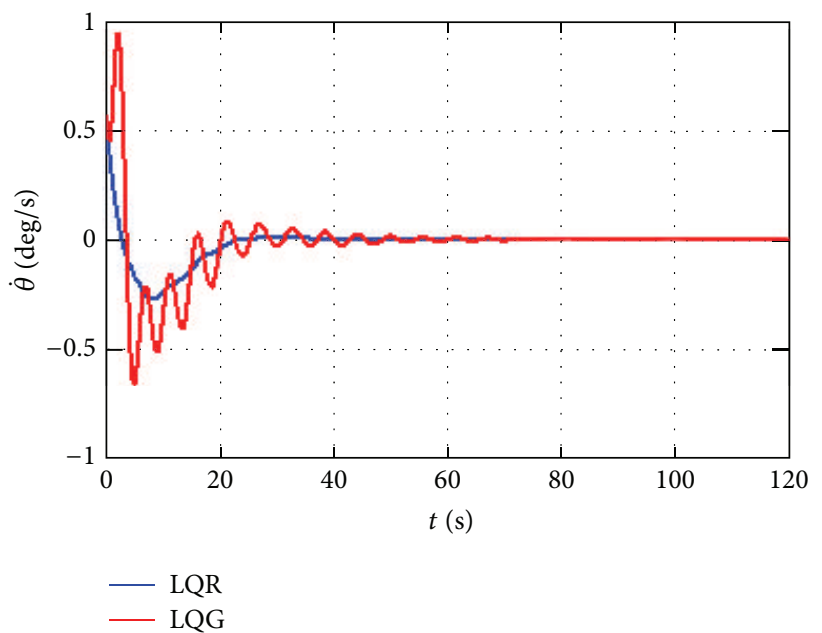

(b)

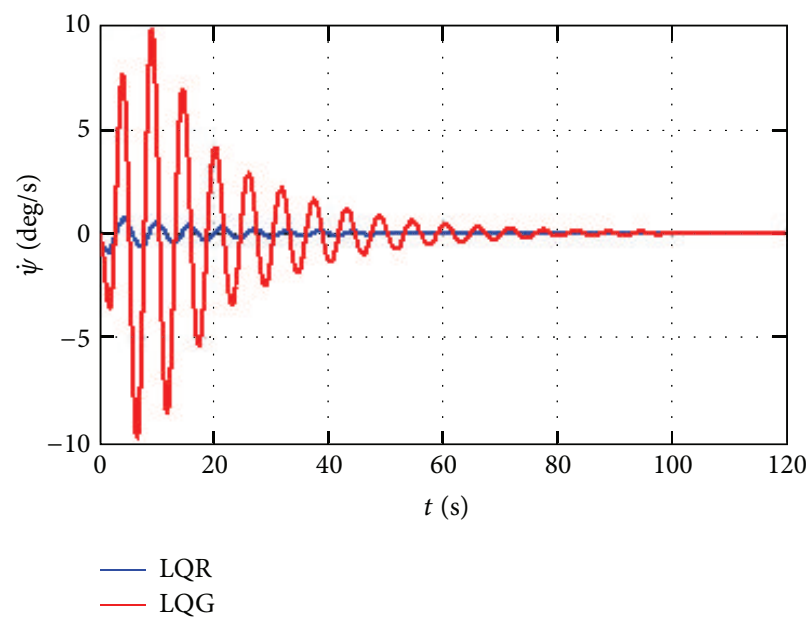

(d)

(c)

FIGURE 3: The angular and sloshing control by the LQR and LQG controller.
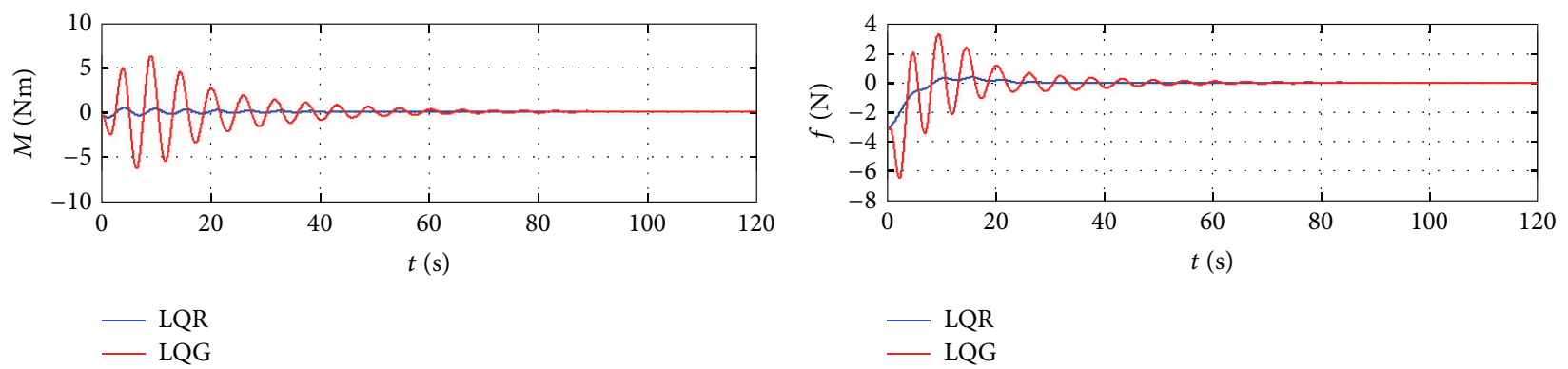

(a)

(b)

FIGURE 4: The LQR and LQG controller effort to control the angular and sloshing motion. 


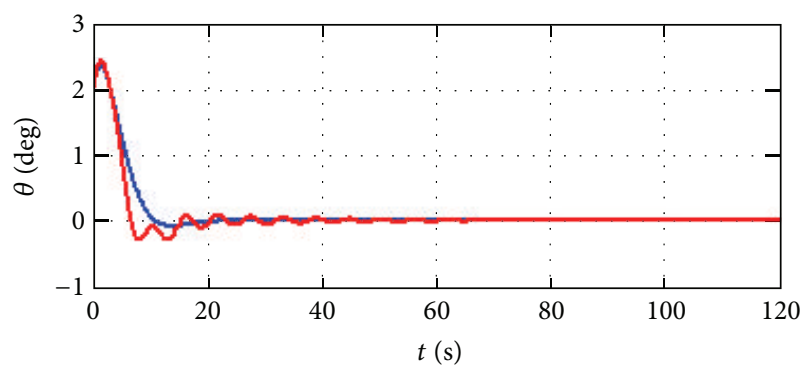

- LQR

— LQG

(a)

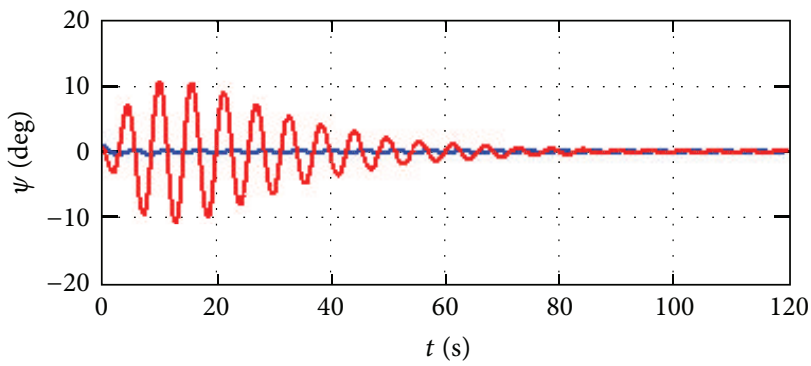

- LQR

— LQG

(c)

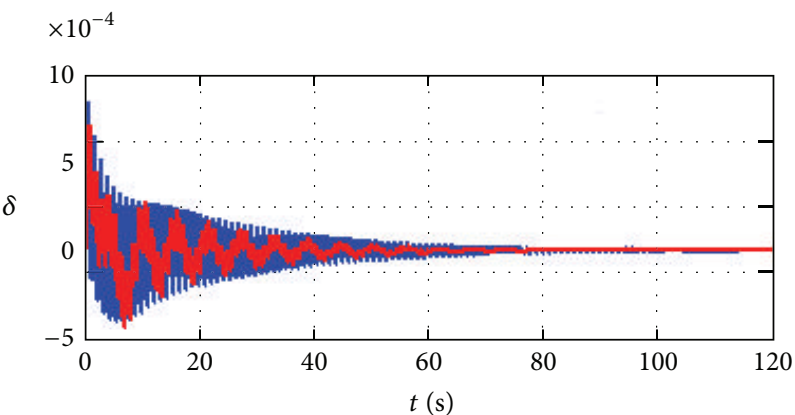

- LQR

— LQG

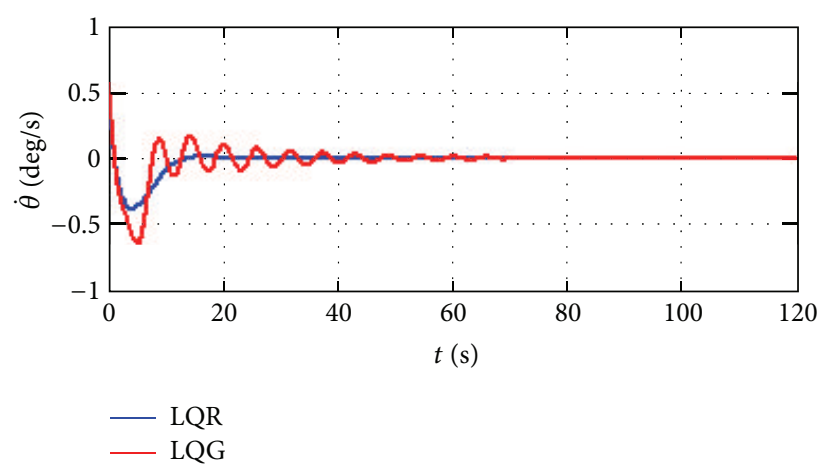

(b)

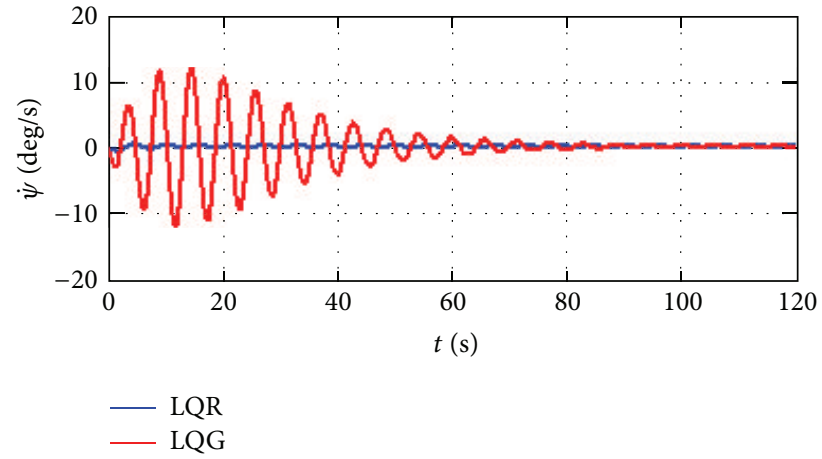

(d)

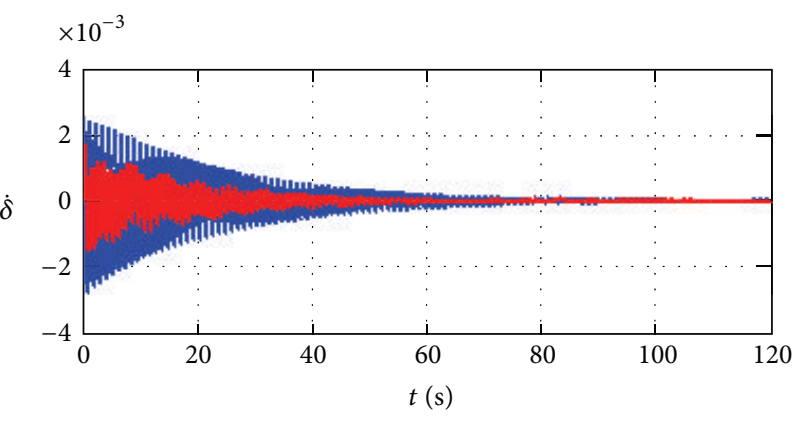

- LQR

- LQG

(e)

(f)

Figure 5: Control of the angular motion, sloshing, and flexibly.

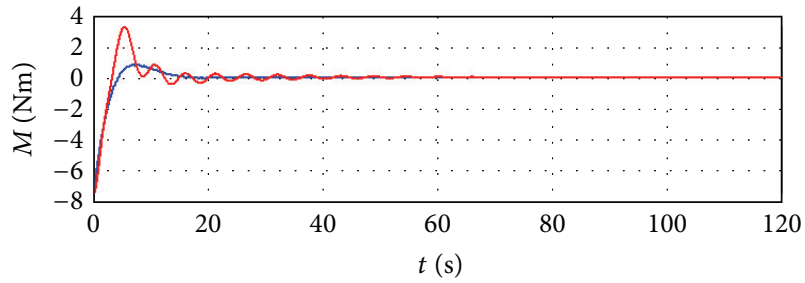

- LQR

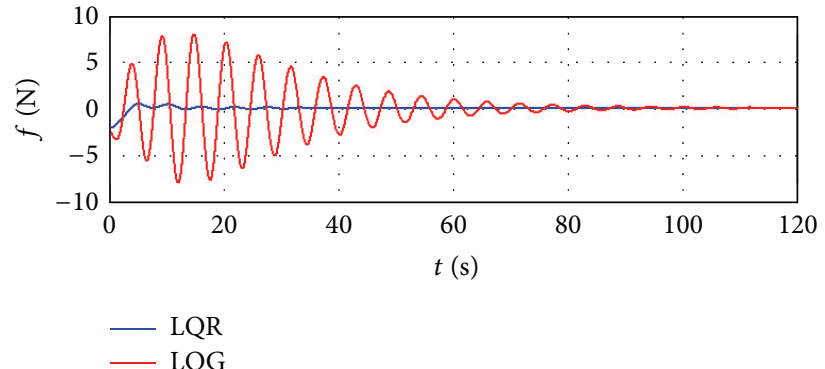

(b)

(a)

, and flexibly. 
Figure 6 shows that both the torque and the force of the LQR controller are smaller than the LQG controller and again this suggests that the LQG controller spends more energy while the control system is estimating the sloshing and flexible variables.

\section{Summary}

In this paper one has developed a new model for the planar dynamics of a rigid-flexible satellite, incorporating the axial, transverse, and pitch dynamics of the satellite, plus fuel slosh dynamics, which is considered underactuated. That model is used to investigate the effects of the interaction between the rigid motion, the liquid motion (slosh), and the flexible satellite dynamics in order to predict what the damage to the controller performance and robustness is. The control inputs are defined by a transverse body fixed force and a moment about the centre of mass. The comparative simulations, designing the satellite ACS by the linear quadratic regulator (LQR) and linear quadratic Gaussian (LQG) methods, have showed a significant improvement in the satellite ACS performance and robustness of what has been done previously, since it simultaneously controls the rigid-flexible satellite motion and the fuel slosh dynamics. But, one observes that the performance of the LQR control is better than and LQG control. The degradation of the LQG control is because this controller spends more energy associated with the force and torque, suggesting that this is due to the estimation process of the sloshing and flexible variables by the Kalman filter. However, this is the realistic situation since both the slosh and the flexible states are not measured for feedback. On the other hand, the LQG controller is more robust than the LQR since it takes into account the noises that represent the imperfections of the models acting over the system. Finally, one observes that flexibility still has small fluctuation, which is not appropriated when one needs high precise pointing. One way to deal with that problem is to apply a nonlinear control technique considering the nonlinear satellite model.

\section{Conflict of Interests}

The authors declare that there is no conflict of interests regarding the publication of this paper.

\section{References}

[1] S. K. Chakrabarti, "Internal waves in a large offshore storage tank," Journal of Energy Resources Technology, vol. 115, no. 2, pp. 134-141, 1993.

[2] D. E. Hill and J. R. Baumgarten, "Control of spin-stabilized spacecraft with sloshing fluid stores," Journal of Dynamic Systems, Measurement and Control, vol. 114, no. 4, pp. 728-731, 1992.

[3] A. Pui-Chun Lui and J. Y. K. Lou, "Dynamic coupling of a liquidtank system under transient excitations," Ocean Engineering, vol. 17, no. 3, pp. 263-277, 1990.

[4] B. N. Agrawal, "Dynamic characteristics of liquid motion in partially filled tanks of a spinning spacecraft," Journal of
Guidance, Control, and Dynamics, vol. 16, no. 4, pp. 636-640, 1993.

[5] M. J. Sidi, Spacecraft Dynamics and Control, Cambridge University Press, New York, NY, USA, 1997.

[6] L. C. G. Souza and R. G. Gonzáles, "Application of the statedependent riccati equation and kalman filter techniques to the design of a satellite control system," Shock and Vibration, vol. 19, no. 5, pp. 939-946, 2012.

[7] L. C. G. Souza, "Design of satellite control system using optimal non-linear theory," Mechanics Based Design of Structures and Machine, vol. 1, pp. 1-20, 2008.

[8] T. P. Sales, D. A. Rade, and L. C. G. de Souza, "Passive vibration control of flexible spacecraft using shunted piezoelectric transducers," Aerospace Science and Technology, vol. 29, no. 1, pp. 403-412, 2013.

[9] I. Mainenti-Lopes, L. C. G. Souza, and F. L. de Sousa, "Design of a nonlinear controller for a rigid-flexible satellite using multi-objective Generalized Extremal Optimization with real codification," Shock and Vibration, vol. 19, no. 5, pp. 947-956, 2012.

[10] L. C .G. Souza and A. G. Souza, "Satellite attitude control system design considering the fuel slosh dynamics," Shock and Vibration, vol. 2014, Article ID 260206, 8 pages, 2014. 


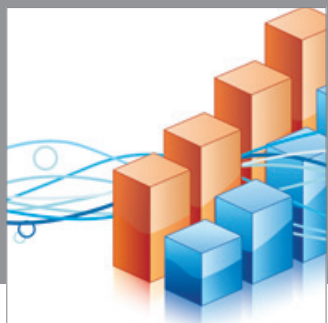

Advances in

Operations Research

mansans

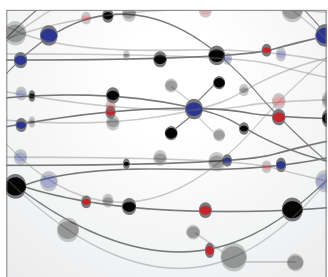

The Scientific World Journal
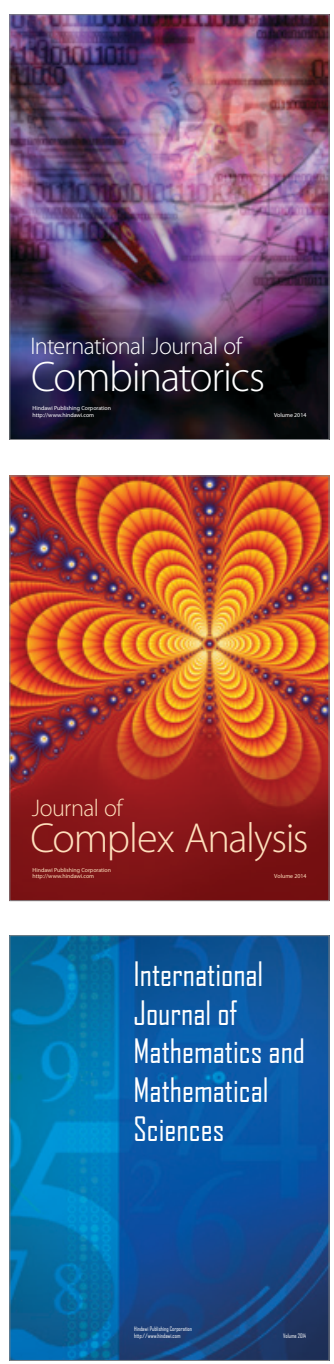
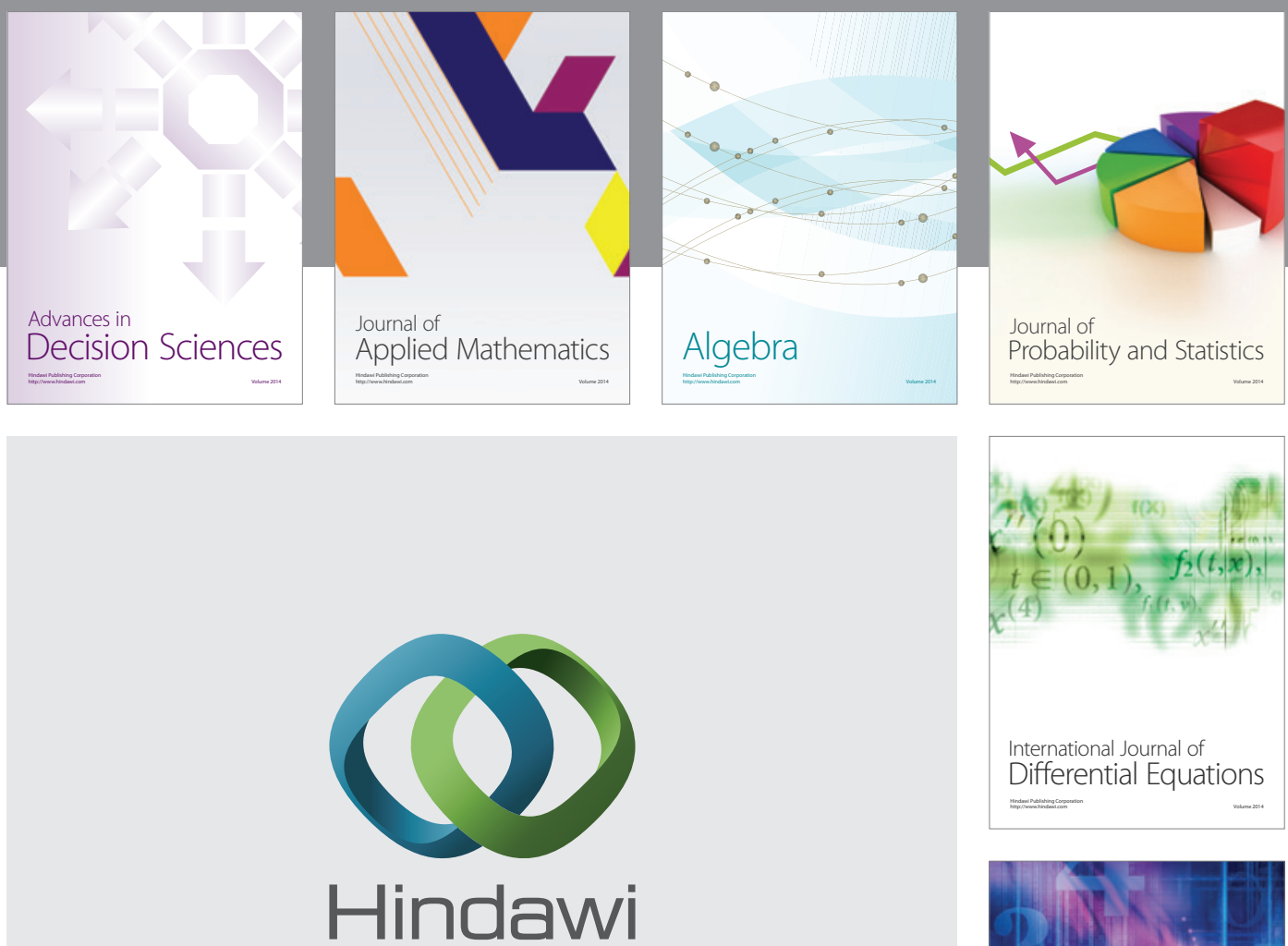

Submit your manuscripts at http://www.hindawi.com
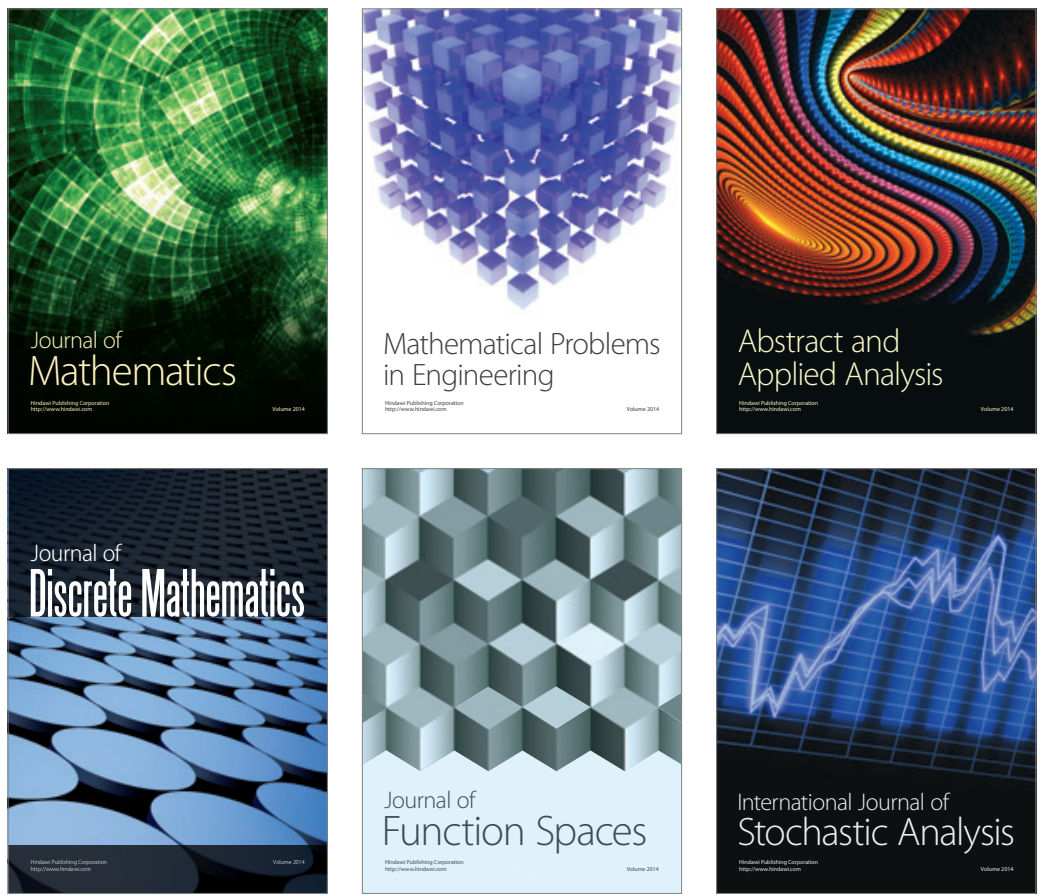

Journal of

Function Spaces

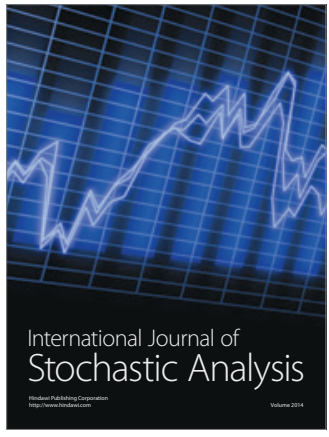

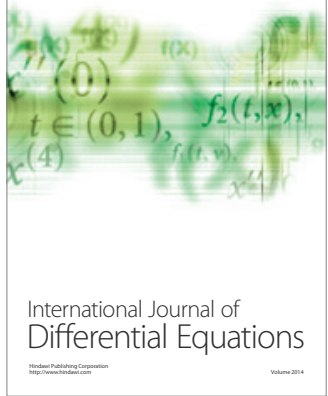
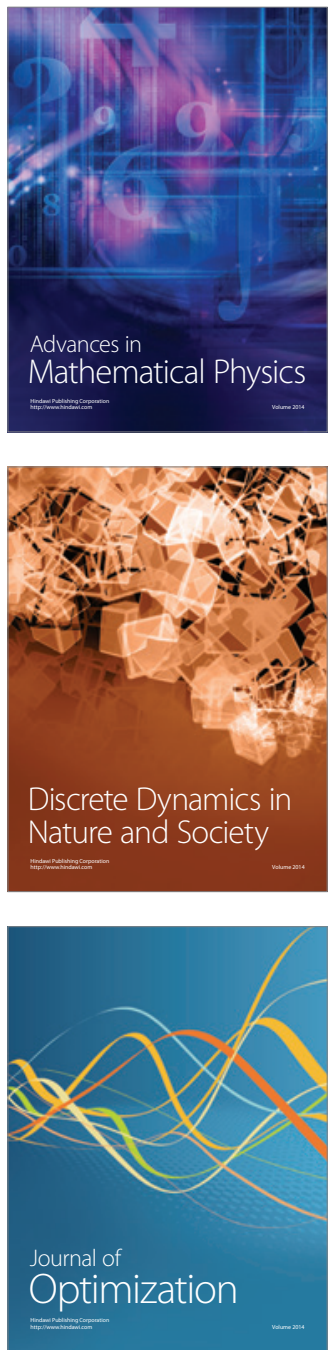